\title{
Improving quality assurance and control
}

Moderator

Bonnie Lawlor 